\title{
Complaints of daytime sleepiness, insomnia, hypnotic use, and risk of dementia: a prospective cohort study in the elderly
}

Clémence Cavaillès ${ }^{1}$, Claudine Berr ${ }^{1}$, Catherine Helmer ${ }^{2}$, Audrey Gabelle ${ }^{1,3}$, Isabelle Jaussent ${ }^{1}$ and Yves Dauvilliers ${ }^{1,4^{*}}$ (1)

\begin{abstract}
Background: Sleep disturbances are common in elderly and occur frequently in dementia. The impact of excessive daytime sleepiness (EDS), insomnia complaints, sleep quality, and hypnotics on the risk of all-cause dementia, Alzheimer disease (AD), and dementia with vascular component (DVC) remains unclear, as does the association between sleep profile and plasma $\beta$-amyloid levels.

Methods: Analyses were carried out on 6851 participants aged 65 years and over randomly recruited from three French cities and free of dementia at baseline. A structured interview and self-questionnaire assessed sleep complaints (EDS, insomnia complaints, sleep quality) and medications at baseline. Incident cases of dementia were diagnosed systematically over a 12-year period. Multivariate Cox models were used to estimate the risk of dementia associated with the sleep complaints considered individually and globally. Plasma $\beta$-amyloid levels were measured by an XMAP-based assay technology in 984 subjects.

Results: After adjustment for socio-demographic characteristics, lifestyle, APOE- $\varepsilon 4$, cardiovascular factors, and depressive status, EDS had a higher risk of all-cause dementia $(H R=1.21 ; 95 \% \mathrm{Cl}=[1.01-1.46])$ and DVC $(H R=1.58$; $95 \% \mathrm{Cl}=[1.07-2.32])$ but not AD. Persistent use of hypnotics increased the risk for all-cause dementia, specifically AD $(\mathrm{HR}=1.28 ; 95 \% \mathrm{Cl}=[1.04-1.58])$, but not DVC. No association was found for insomnia complaints and sleep quality taken as individual factors or combined with EDS on the risk of dementia. No association was found between $\beta$-amyloid, sleep complaints, and incident dementia.
\end{abstract}

Conclusions: The results suggest a deleterious role of EDS and hypnotics on dementia. Further studies are required to elucidate the mechanisms involved in these associations and whether its management can prevent the risk of dementia.

Keywords: Sleepiness, Hypnotic use, Insomnia complaints, Dementia, Alzheimer, Elderly, Cohort studies, Epidemiology

*Correspondence: ydauvilliers@yahoo.fr

${ }^{4}$ National Reference Centre for Orphan Diseases, Narcolepsy- Rare Hypersomnias, Sleep Unit, Department of Neurology, CHU Montpellier, Service de Neurologie, Hôpital Gui-de-Chauliac, 80 avenue Augustin Fliche, 34295 Montpellier cedex 5, France

Full list of author information is available at the end of the article

\section{Introduction}

The burden of dementia is important and continues to increase [1]. Since no pharmacological treatment has shown to be effective, the need to prevent dementia is a major public health issue. An increasing attention has been paid to sleep recently. Aging in the general adult population is associated with an increase of sleep original author(s) and the source, provide a link to the Creative Commons licence, and indicate if changes were made. The images or other third party material in this article are included in the article's Creative Commons licence, unless indicated otherwise in a credit line to the material. If material is not included in the article's Creative Commons licence and your intended use is not permitted by statutory regulation or exceeds the permitted use, you will need to obtain permission directly from the copyright holder. To view a copy of this licence, visit http://creativecommons.org/licenses/by/4.0/. The Creative Commons Public Domain Dedication waiver (http://creativeco mmons.org/publicdomain/zero/1.0/) applies to the data made available in this article, unless otherwise stated in a credit line to the data. 
problems particularly insomnia and excessive daytime sleepiness (EDS) which have been associated with a higher risk of all-cause cognitive decline or dementia [2-11]. However, large heterogeneities in the results were observed and could be attributed to multifactorial nature and cause of sleep disturbances, differences in sleep and cognitive assessments, use of hypnotics, study design, duration of follow-up, and sample size $[2,3,5,11-13]$. A recent large-scale study of only men showed that sleep problems defined as difficulties falling asleep, waking up in the early hours, or just taking sleeping pills were associated with dementia incidence, specifically Alzheimer' disease (AD) [8]. However, the specific role of these three complaints was not studied, and the impact of EDS was not evaluated. Furthermore, the impact of sleep disturbances may depend on the etiologies of dementia associated with different pathophysiologies. Dementia and particularly $\mathrm{AD}$ is mainly characterized by excessive production and/or cleavage of amyloid precursor protein and by intra-neuronal hyperphosphorylated and aggregated tau proteins with altered brain clearance. In contrast, although with possible overlap with $\mathrm{AD}$, dementia with vascular component (DVC) is primarily associated with comorbid cardiovascular conditions, as well as hypoxia, inflammation, and oxidative stress. Yet, few studies have investigated the associations between sleep disturbances and the type of dementia and showed that insomnia was often predictive of all-cause dementia and $\operatorname{AD}[7,8,10]$, and EDS of DVC [3].

As demographic and clinical characteristics may be associated with both sleep problems and dementia, it remains important to determine whether certain populations (e.g., old age, women, carriers of apolipoprotein e- $\varepsilon 4$ (APOE- $\varepsilon 4$ ), cardiometabolic diseases, or depression) may modify the associations between sleep complaints and dementia risk. It is also unknown whether there is a synergistic effect of sleep problems on the risk of cognitive disorders. A more complete assessment of sleep problems and their severity may clarify their potential additive roles on the risk of dementia.

We thus evaluated the association between the use of hypnotics, sleep complaints (EDS, insomnia complaints, and sleep quality) analyzed separately as individual dimensions and combined together in an overall assessment, and the risk of dementia (all-cause, AD, and DVC) over 12 years in the elderly. We also studied if specific populations modify the association between sleep problems, hypnotic intake, and dementia risk and deciphered the relationship between sleep profile and plasma $\beta$-amyloid concentrations.

\section{Methods}

\section{Study population}

This study was part of the Three-City Study [14], a longitudinal cohort conducted in three French cities: Bordeaux $(n=2104)$, Dijon $(n=4931)$, and Montpellier $(n$ $=2259$ ). Participants were non-institutionalized subjects, aged 65 years or over, and registered on the electoral rolls. Its main objective was to estimate the risk of dementia attributable to vascular factors. Overall, 9294 participants were recruited from the electoral rolls between 1999 and 2001. The participants were interviewed and underwent clinical examinations at baseline and after 2, 4, 8, 10, and 12 years.

\section{Dementia diagnosis}

Dementia was screened and diagnosed at baseline and at each follow-up visit according to DSM-IV revised criteria and further validated independently by a national panel of neurologists. The onset of dementia was estimated at the midpoint between diagnosis and the prior examination without dementia. Etiology was based on all available information (e.g., neuropsychological test, daily activities, neurological examination, and hospitalization records), on the National Institute of Neurological and Communicative Disorders and Stroke-Alzheimer's Disease and Related Disorders Association criteria for Alzheimer disease (AD) and, the National Institute of Neurological Disorders and Stroke-Association Internationale pour la Recherche et l'Enseignement en Neurosciences criteria for vascular dementia $[15,16]$. The causes of dementia were divided into three types: AD, DVC (including pure vascular dementia and mixed dementia due to a small number of cases), and other dementias (including dementia with Lewy bodies, Parkinson's dementia, fronto-temporal dementia [17-20].

\section{Sleep complaints and medication}

Current sleep problems were self-reported at baseline by the completion of a specific questionnaire including 5 items: "How would you assess your sleep?" (sleep quality (SQ)); "Do you feel very sleepy during the day?" (EDS); "Do you have any difficulty in falling sleep?" (difficulty in initiating sleep (DIS)); "Do you wake-up during the night?" (difficulty maintaining sleep (DMS)); "Do you often wake-up early in the morning without being able to go back to sleep?" (early morning awakening (EMA)). The questions were answered on a 4-point Likert scale $(0=$ never, $1=$ rarely, $2=$ frequently, $3=$ often) except for $S Q$ rated on 3 -point $(0=$ good, $1=$ average, $2=$ bad $)$. Sleep complaints were first examined as individual dimensions.

The presence of EDS, DIS, DMS, and EMA was defined as reporting the related complaint "frequently" or "often," 
and a poor SQ as reporting "average" or "poor" SQ to allow a sufficient number of dementia cases in each sleep category.

As a second step, we proposed to combine together the sleep complaints (EDS, insomnia complaints, and sleep quality) in a clinical sleep severity (CSS) score to estimate their potential additive role on the risk of dementia. CSS score was defined by the sum of the 5 -item responses. To standardize the response choice, the SQ item was recoded as $0=$ good, $1.5=$ average, and $3=$ bad. A total score ranged from 0 to 15 with a higher score indicated more severe sleep problems. It was studied as a continuous variable but also as a categorical variable according to the tertiles of the score distribution in the whole sample.

At each visit, the use of prescribed drugs during the preceding month was checked by the interviewer. Current use of medication was based the World Health Organization Anatomical Therapeutic Chemical (ATC) classification system. Hypnotic intake was defined as taking at least one benzodiazepines (BZD) or BZD-like compounds (zolpidem, zopiclone), or miscellaneous medications (including barbiturates, antihistaminics, and other pharmacological categories such as neuroleptics and sedative antidepressants (e.g., doxepin, mirtazapine, trazodone)) during the preceding month.

\section{Other variables measured at inclusion}

Standardized evaluation included questions related to demographic characteristics, educational level $(<6$ years; [6-12] years; $\geq 12$ years), living status (alone or not), daily life habits such as alcohol consumption $(<12 \mathrm{~g} /$ day; [12;36]; $\geq 36)$, caffeine consumption ( $\leq 125 \mathrm{mg} /$ day; [125;375]; > 375), smoking status (none; former; current smoker), and mobility (no restrictions to move; being confined to bed, seat, home, or neighborhood). Health status included body-mass index (BMI) $(<25$; [25-30]; $\geq 30 \mathrm{~kg} / \mathrm{m}^{2}$ ), hypertension (current antihypertensive treatment or systolic/diastolic blood pressure $>160 / 95$ $\mathrm{mmHg}$ ), diabetes (treatment with antidiabetic agents or fasting glucose level $\geq 7.0 \mathrm{mmol} / \mathrm{L}$ ), hypercholesterolemia (treatment with lipid-lowering agents or total cholesterol level $\geq 6.2 \mathrm{mmol} / \mathrm{L}$ ), and cardio-cerebrovascular diseases (angina pectoris, myocardial infarction, cardiovascular surgery, arteritis, and stroke). Depressive status was defined as a score $\geq 16$-point on the Center for Epidemiological Studies-Depression Scale or current antidepressant treatment. APOE- $\varepsilon 4$ was genotyped as described previously [21]. Non-fasting plasma $\beta$-amyloid peptide $40\left(\mathrm{~A} \beta_{40}\right)$ and $42\left(\mathrm{~A} \beta_{42}\right)$ samples were collected at baseline in a subgroup in tubes containing salt ethylenediaminetetra-acetic as an anticoagulant. Following centrifugation, plasma samples were aliquoted into polypropylene tubes, stored at $-80^{\circ} \mathrm{C}$ and only thawed immediately prior to $\beta$-amyloid quantification. Plasma $\beta$-amyloid peptide levels were measured blind to cognitive status. The plasma $\beta$-amyloid peptide assay was performed using the INNO-BIA kit (Innogenetics, Ghent, Belgium), based on a multiplex xMAP technique (Luminex, Austin, TX) [22, 23].

\section{Statistical analyses}

Cox models on complete data with delayed entry and age of the participants as the time scale were used to estimate the hazard ratios (HR) and their 95\% confidence intervals (CI) for the associations between sleep complaints, hypnotic use, and the incidence of all-cause dementia over the 12-year follow-up. Several multivariable models including study center, age, sex, and potential confounders selected from the literature and associated with all-cause dementia in univariate models $(p<0.15)$ were successively performed. Model 1 was adjusted for study center, age, sex, education, mobility, and APOE- $\varepsilon 4$ allele. Model 2 was further adjusted for cardiovascular and metabolic diseases, and model 3 was further adjusted for depressive status. The proportional hazards assumption was tested using the Schoenfeld residuals. In the presence of non-proportionality, stratification on the dependent variable was applied. Incident AD and DVC were further analyzed as distinct end-points. Additional sensitivity analyses were performed to ensure the robustness of the results and to address possible reverse causality: (1) adjustment for hypnotic use, (2) exclusion of participants using hypnotics, (3) imputation of missing data on covariates in 539 participants using Multiple Imputation Chained Equation (7\% of missing data, 10 imputation templates were generated), and (4) exclusion of dementia cases at a 2-year follow-up. In supplementary analyses, time-dependent Cox models were performed to take into account the use of hypnotics during the follow-up. The use of hypnotic was considered to be a time-dependent variable, with data for hypnotics use defined throughout follow-up as: no user (no hypnotic during the follow-up), intermittent users (cumulative proportion of hypnotic use $\leq 50 \%$ over the entire follow-up), and persistent users (cumulative proportion of hypnotic use $>50 \%$ over the entire follow-up). Interactions between significant sleep complaints, hypnotic use, and several factors describing population at higher risk for dementia (i.e., age, sex, depressive status, APOE- $\varepsilon 4$, history of cardiovascular, and metabolic diseases) on dementia risk were tested using the Wald- $\chi^{2}$ test for all-cause dementia, AD, and DVC.

In a subsample, Cox models were performed to study the association between plasma $A \beta$ levels and incident dementia. Linear regressions were used to study the association between sleep complaints, hypnotics intake, 
and $\beta$-amyloid levels (taken as a continuous variable or categorized into tertiles). Significance level was set at a two-sided $p<0.05$. Statistical analyses were performed using SAS 9.4 (SAS Institute Inc.) and R 3.6.3 statistical software.

\section{Results}

\section{Subjects characteristics}

As shown in the study diagram (Fig. 1), the study sample included 6851 participants free of dementia $(60 \%$ women) with a mean age of 73.7 years $(\mathrm{SD}=5.3)$, with completed sleep questionnaires, with follow-up, and no missing data on covariates. Subjects excluded from the study were more frequently female, older, had a lower education level, living alone, and were more often confined, and present more metabolic and cardiovascular diseases, depressive status, and sleep complaints.

Regarding baseline sleep characteristics, the presence of EDS, DIS, DMS, and EMA was reported by $18.1 \%$, $35.3 \%, 63.7 \%$, and $36.8 \%$ of the participants respectively, while the presence of a poor SQ was reported by $52.6 \%$ of the participants. Around a quarter (23\%) reported no sleep complaints, $25 \%$ one complaint, $50 \%$ two to four complaints, and only $2 \%$ had the five complaints. A higher CSS score was found older adults and in subjects with more cardiovascular diseases and depressive status.

Hypnotics at baseline were taken by $22.5 \%$ of subjects: 16.2\% BZD, 5.0\% BZD-like compounds, 1.9\% antihistaminic compounds, $1.4 \%$ sedative antidepressants, and $1.2 \%$ other medications. Among these subjects treated with hypnotics at baseline, $28.2 \%$ had a poor SQ, $21.5 \%$ EDS, 57.4\% DIS, 70.2\% DMS, and 51.3\% EMA. Compared to participants without hypnotics use, they were more likely to have increased EDS and insomnia complaints, a poorer SQ, and a higher CSS score $(p<0.0001$, for all comparisons).

\section{Associations between sleep complaints, hypnotic intake, and incident dementia over the 12-year follow-up}

After a median follow-up of 8.9 years [IQR $=3.9-11.2$ years], 695 participants were diagnosed with dementia: 470 with AD, 139 with DVC, and 86 with other dementias. Compared to participants free of dementia, those with incident all-cause dementia had more frequently a lower educational level, diabetes mellitus, cardiovascular

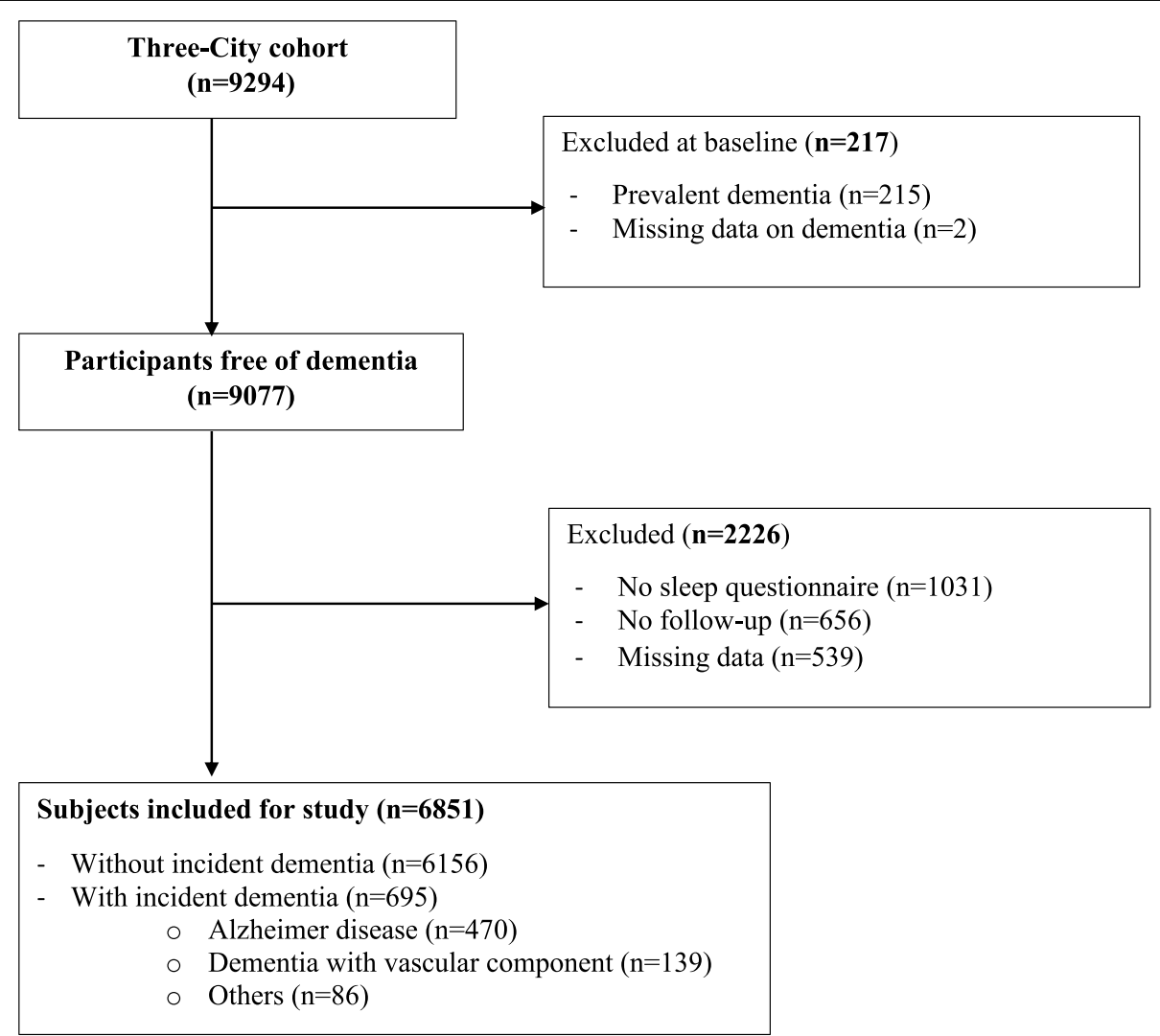

Fig. 1 Flow chart of the sample. This figure represents the sample selection for the analysis of excessive daytime sleepiness, insomnia complaints and hypnotic use, and the risk of dementia 
Table 1 Baseline demographic and clinical characteristics of 6851 participants according to all-cause dementia over a 12-year follow-up

\begin{tabular}{|c|c|c|c|c|c|c|c|c|}
\hline \multirow[t]{2}{*}{ Variables } & \multicolumn{2}{|c|}{$\begin{array}{l}\text { Whole sample, } n= \\
6851\end{array}$} & \multicolumn{2}{|c|}{$\begin{array}{l}\text { Free of dementia, } n \\
=6156\end{array}$} & \multicolumn{2}{|c|}{$\begin{array}{l}\text { Incident dementia, } \\
n=695\end{array}$} & \multirow[t]{2}{*}{$\mathrm{HR}[95 \% \mathrm{Cl}]$} & \multirow[t]{2}{*}{$p$ value } \\
\hline & $n$ & $\%$ & $n$ & $\%$ & $n$ & $\%$ & & \\
\hline Sex, women & 4118 & 60.11 & 3672 & 59.65 & 446 & 64.17 & $1.03[0.89-1.21]$ & 0.67 \\
\hline Education (years) & & & & & & & & $<0.0001$ \\
\hline$<6$ & 1583 & 23.11 & 1356 & 22.03 & 227 & 32.66 & 1 & \\
\hline$[6 ; 11]$ & 2484 & 36.26 & 2276 & 36.97 & 208 & 29.93 & $0.57[0.47-0.69]$ & \\
\hline$\geq 12$ & 2784 & 40.64 & 2524 & 41.00 & 260 & 37.41 & $0.61[0.51-0.73]$ & \\
\hline Live alone, yes & 2338 & 34.13 & 2062 & 33.50 & 276 & 39.71 & $1.00[0.85-1.16]$ & 0.97 \\
\hline BMI $\left(\mathrm{kg} / \mathrm{m}^{2}\right)$ & & & & & & & & 0.17 \\
\hline$<25$ & 3280 & 47.88 & 2959 & 48.07 & 321 & 46.19 & 1 & \\
\hline$[25 ; 30]$ & 2683 & 39.19 & 2399 & 38.97 & 284 & 40.86 & $1.13[0.96-1.32]$ & \\
\hline$\geq 30$ & 888 & 12.96 & 798 & 12.96 & 90 & 12.95 & $1.21[0.96-1.53]$ & \\
\hline Smoking status, current/past & 2692 & 39.29 & 2442 & 39.67 & 250 & 35.97 & $0.99[0.85-1.16]$ & 0.92 \\
\hline Mobility, confined & 352 & 5.14 & 279 & 4.53 & 73 & 10.50 & $1.98[1.54-2.53]$ & $<0.0001$ \\
\hline Alcohol (g/day) & & & & & & & & 0.45 \\
\hline$<12$ & 4423 & 64.56 & 3959 & 64.31 & 464 & 66.76 & 1 & \\
\hline$[12 ; 36[$ & 1835 & 26.78 & 1663 & 27.01 & 172 & 24.75 & $0.91[0.77-1.09]$ & \\
\hline$\geq 36$ & 593 & 8.66 & 534 & 8.67 & 59 & 8.49 & $1.08[0.83-1.42]$ & \\
\hline Caffeine (mg/day) & & & & & & & & 0.79 \\
\hline$\leq 125$ & 1767 & 25.79 & 1577 & 25.62 & 190 & 27.34 & 1 & \\
\hline ]125; 375] & 4029 & 58.81 & 3629 & 58.95 & 400 & 57.55 & $0.95[0.80-1.13]$ & \\
\hline$>375$ & 1055 & 15.40 & 950 & 15.43 & 105 & 15.11 & $1.00[0.79-1.27]$ & \\
\hline Hypercholesterolemia, yes & 3888 & 56.75 & 3483 & 56.58 & 405 & 58.27 & $1.11[0.95-1.29]$ & 0.19 \\
\hline Diabetes mellitus, yes & 613 & 8.95 & 516 & 8.38 & 97 & 13.96 & $1.89[1.52-2.34]$ & $<0.0001$ \\
\hline Hypertension, yes & 4080 & 59.55 & 3640 & 59.13 & 440 & 63.31 & $1.06[0.91-1.24]$ & 0.46 \\
\hline History of cardiovascular disorders, yes & 1882 & 27.47 & 1660 & 26.97 & 222 & 31.94 & $1.17[1.00-1.38]$ & 0.05 \\
\hline Depressive status ${ }^{\mathrm{a}}$, yes & 1726 & 25.19 & 1482 & 24.07 & 244 & 35.11 & $1.67[1.43-1.95]$ & $<0.0001$ \\
\hline Carrier of the APOE- $\varepsilon 4$ allele, yes & 1346 & 19.65 & 1151 & 18.70 & 195 & 28.06 & $1.98[1.68-2.34]$ & $<0.0001$ \\
\hline \multicolumn{9}{|l|}{ In a subsample of 984 subjects } \\
\hline$A \beta_{40}(p g / m l)$ & & & & & & & & 0.91 \\
\hline$<214.31$ & 328 & 33.33 & 295 & 33.26 & 33 & 34.02 & 1 & \\
\hline$[214.31 ; 251.96]$ & 328 & 33.33 & 297 & 33.48 & 31 & 31.96 & $0.92[0.56 ; 1.50]$ & \\
\hline$>251.96$ & 328 & 33.33 & 295 & 33.26 & 33 & 34.02 & $0.91[0.56 ; 1.47]$ & \\
\hline$A \beta_{42}(p g / m l)$ & & & & & & & & 0.09 \\
\hline$<33.69$ & 326 & 33.23 & 292 & 33.03 & 34 & 35.05 & 1 & \\
\hline$[33.69 ; 42.30]$ & 329 & 33.54 & 289 & 32.69 & 40 & 41.24 & $1.15[0.73 ; 1.81]$ & \\
\hline$>42.30$ & 326 & 33.23 & 303 & 34.28 & 23 & 23.71 & $0.65[0.38 ; 1.10]$ & \\
\hline Ratio $A \beta_{42} / A \beta_{40}$ & & & & & & & & 0.24 \\
\hline$<0.150$ & 330 & 33.64 & 293 & 33.14 & 37 & 38.14 & 1 & \\
\hline$[0.150 ; 0.182]$ & 319 & 32.52 & 284 & 32.13 & 35 & 36.08 & $1.01[0.63 ; 1.60]$ & \\
\hline$>0.182$ & 332 & 33.84 & 307 & 34.73 & 25 & 25.77 & $0.68[0.41 ; 1.12]$ & \\
\hline
\end{tabular}

${ }^{a}$ Center for Epidemiological Studies-Depression Scale $\geq 16$ or current antidepressant treatment

disorder, and depressive status and were more likely to be confined at home and carriers of APOE- $\varepsilon 4$ (Table 1).

EDS was associated with incident all-cause dementia even after adjustment for cardiovascular comorbidities (Table 2, model 2) or further adjustment for depressive status (Table 2, model 3). EDS was also associated with incident DVC but no association was observed for AD patients (Tables 3 and 4). No association was found between DIS, DMS, EMA, SQ, and CSS score and either all-cause dementia incidence (Table 2) or the subtypes 


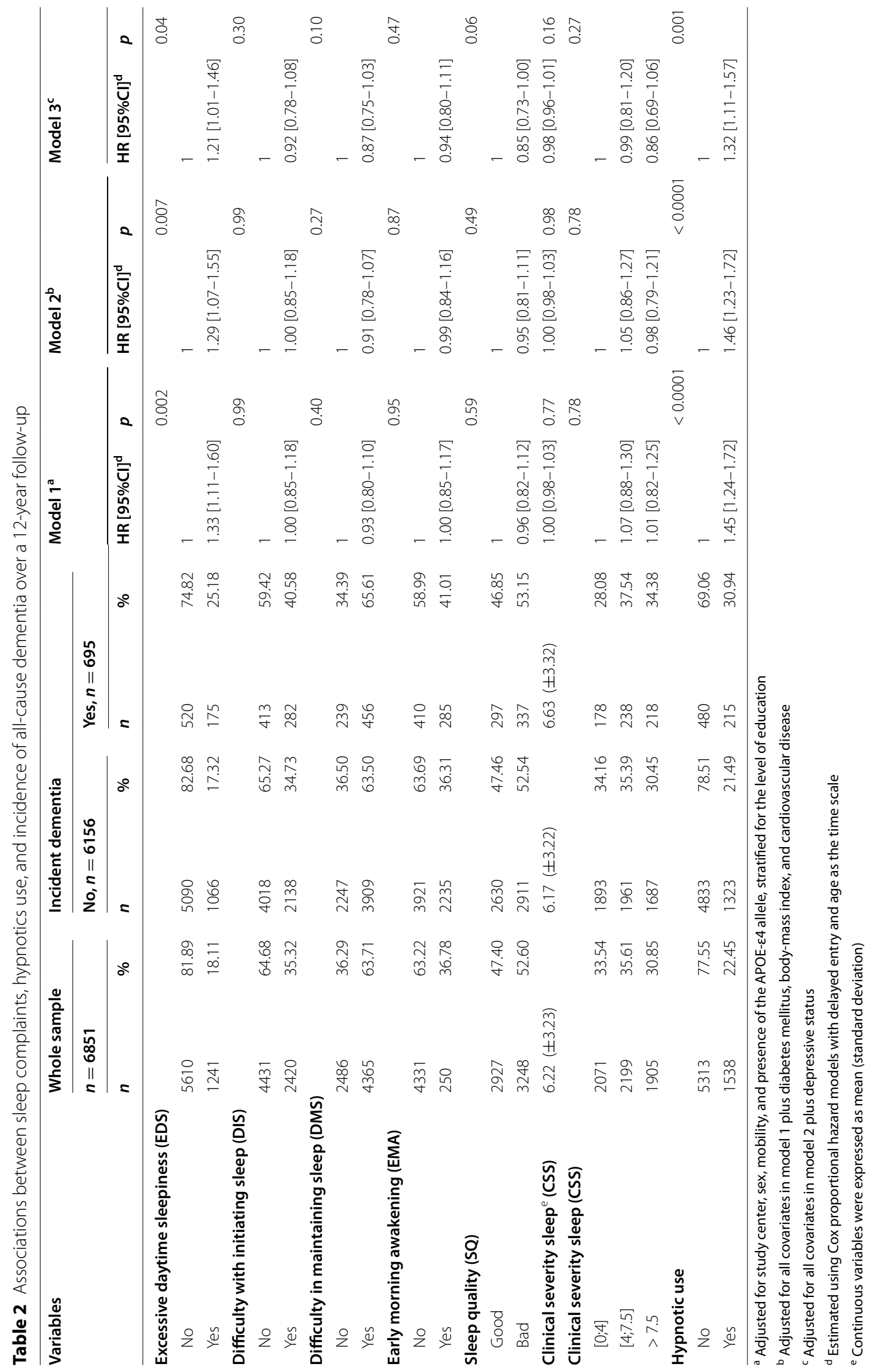




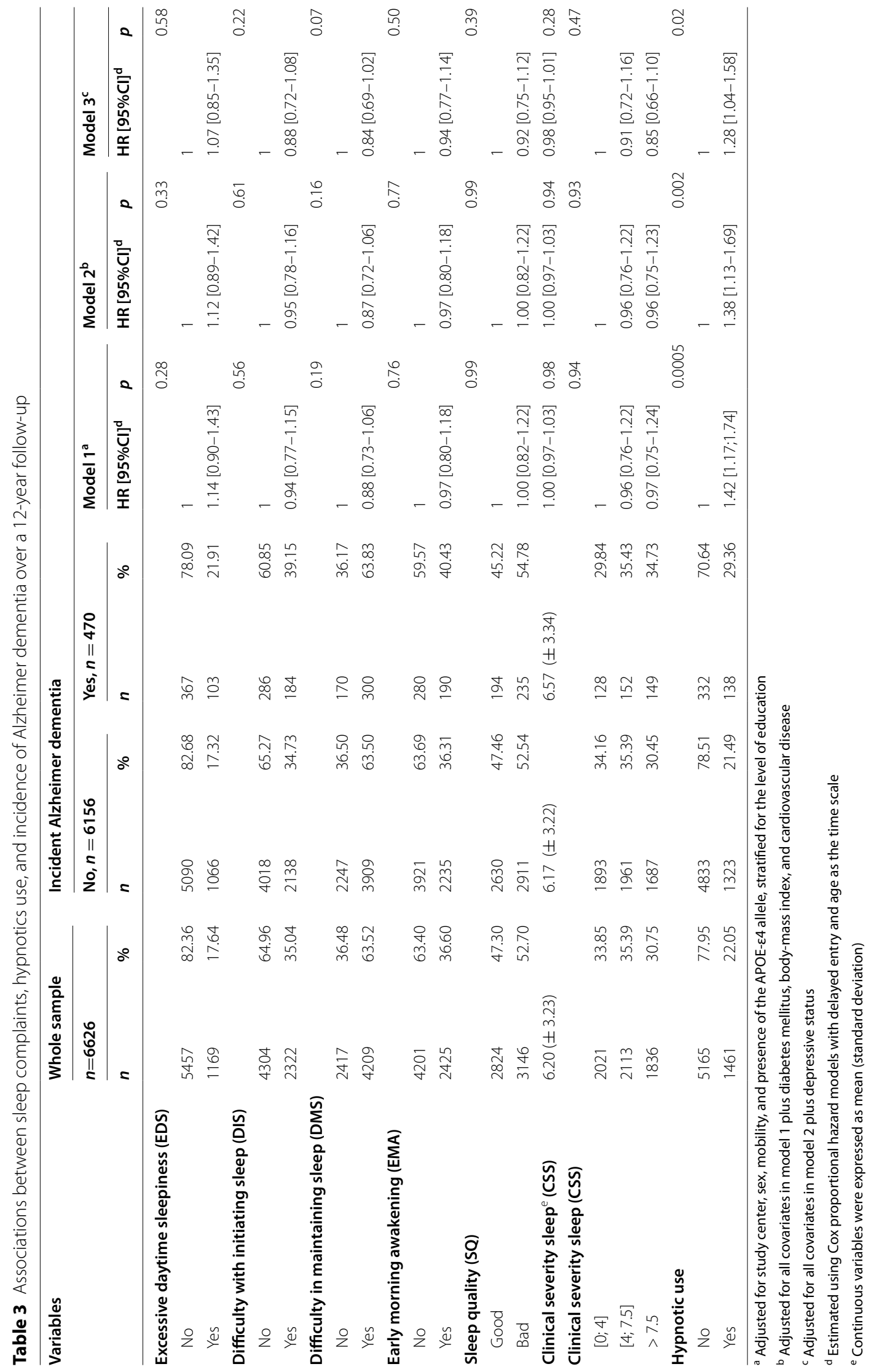




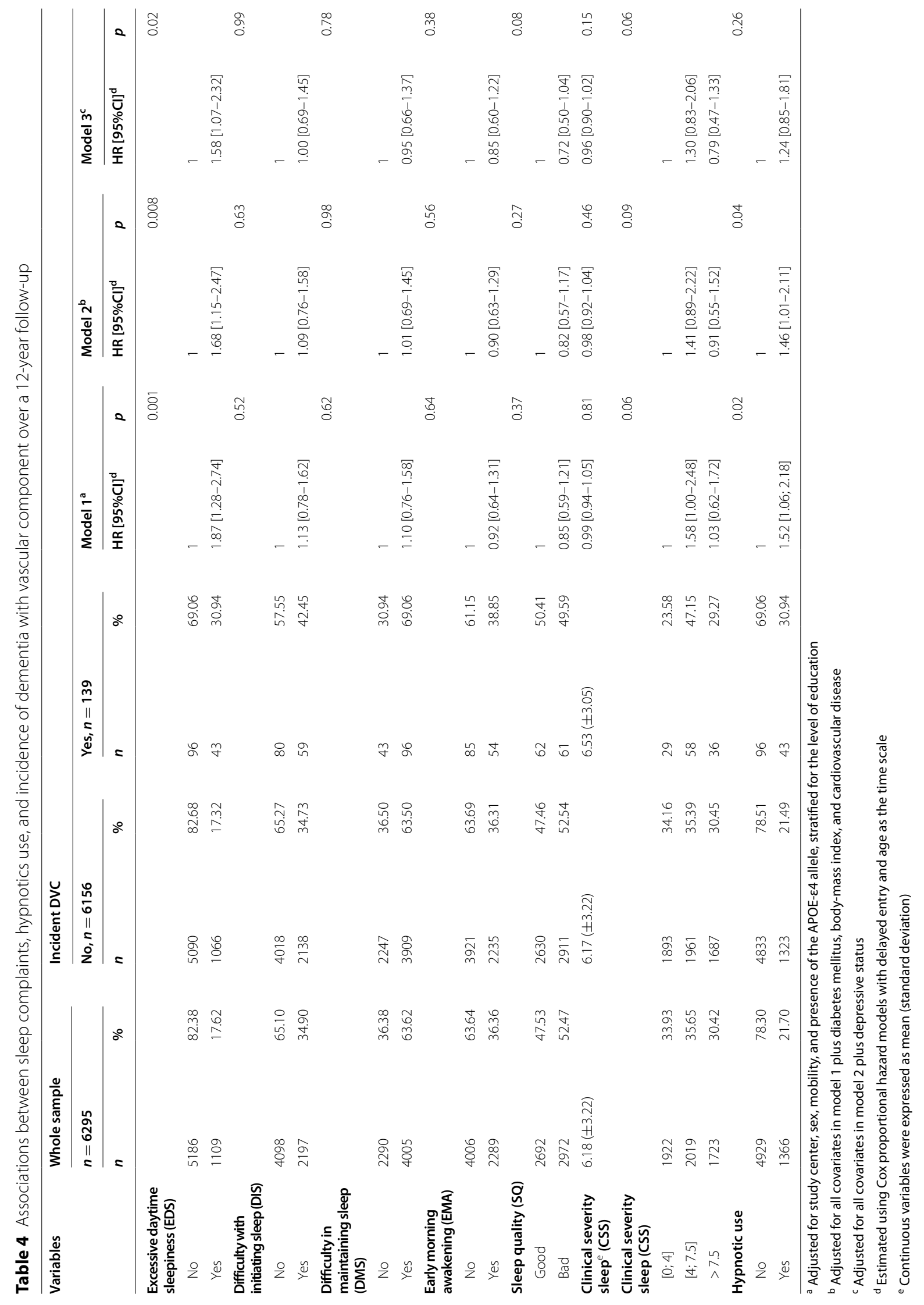


of dementia (Tables 3 and 4). All these results remained unchanged after adjustment for hypnotic intake or excluding participants taking hypnotics (analyses on 5313 subjects (of whom $332 \mathrm{AD}$ and 96 DVC)). Imputations of the $7 \%$ of missing covariates data (Fig. 1) yielded also consistent results with the main analysis $(n=7390$ (of whom $522 \mathrm{AD}$ and $157 \mathrm{DVC}$ )) as well as excluding incident dementia on the first 2-year follow-up (analyses on 6752 subjects (of whom $403 \mathrm{AD}$ and $119 \mathrm{DVC}$ )).

The use of hypnotics at baseline, particularly BZD (72\% of sleep medication), was independently associated with a higher risk of all-cause dementia, $\mathrm{AD}$, and DVC even after several adjustments (Tables 2, 3, and 4, model 2). When further adjusting for depressive status, the association was only significant for all-cause dementia and AD (Tables 2, 3, and 4, model 3).

In supplementary analyses, we examined the association between persistent use of hypnotics and the risk of dementia in two different ways. First, we studied the relationship between the use of hypnotics during the first 2 years and the risk of dementia. A total of 4454 subjects (68.3\%) did not report hypnotic use at baseline and at 2 years of follow-up, 1203 (18.5\%) reported using both at baseline and at 2 years (persistent users), and 865 (13.3\%) were taking hypnotics at one of two time points (intermittent users). The risk of all-cause dementia for the next 10 years was associated with the persistent use of hypnotics ( $\mathrm{p}$-global $=0.009$, compared to non-users, $\mathrm{HR}=$ $1.20,95 \% \mathrm{CI}=[0.94-1.53]$ for intermittent users; HR $=$ $1.37,95 \% \mathrm{CI}=[1.12-1.68]$ for persistent users; model 3$)$. Similar results were found for AD ( $\mathrm{p}$-global $=0.04$, compared to non-users, $\mathrm{HR}=1.10,95 \% \mathrm{CI}=[0.81-1.49]$ for intermittent users; $\mathrm{HR}=1.39,95 \% \mathrm{CI}=[1.08-1.79]$ for persistent users). No association was found between the use of hypnotics and incident DVC. Second, we reported the use of hypnotics throughout the follow-up, with 4029 (58.8\%) non-user, 1488 (21.7\%) persistent users, and 1334 (19.5\%) intermittent users. Compared to non-user, persistent users were associated with dementia ( $\mathrm{HR}=$ $2.15,95 \% \mathrm{CI}=[1.76-2.63]$; model 3) while there was no association for intermittent users $(\mathrm{HR}=0.99,95 \% \mathrm{CI}=$ [0.82-1.19]; model 3).

\section{Interaction between EDS, hypnotic intake, and factors describing specific populations with regard to dementia incidence}

Association between hypnotic use at baseline and incident all-cause dementia differed according to diabetes status ( $p=0.03$ for interaction test) with a higher risk in diabetic subjects $(\mathrm{HR}=2.22,95 \% \mathrm{CI}=[1.47-3.37])$ compared to non-diabetics $(\mathrm{HR}=1.36,95 \% \mathrm{CI}=[1.13-$ 1.62]). Similar results were found for incident AD but not DVC. No interaction was found between EDS, hypnotic use, and factors describing specific populations (i.e., sex, depressive status, APOE- $\varepsilon 4$, history of metabolic, and cardiovascular diseases) on the risk of all-cause dementia and subtypes.

The sample was also stratified according to age ( $\geq 75$ years (median of the whole sample) vs $<75$ years). EDS was associated with all-cause dementia incidence in the older group after adjustments $(\mathrm{HR}=1.29,95 \% \mathrm{CI}=$ [1.02-1.64]) but not in the youngest group ( $\mathrm{HR}=1.11$, $95 \% \mathrm{CI}=[0.82-1.49])$. Results were unchanged for DVC, but no association was found between EDS and AD in any age group. The use of hypnotics at baseline was associated with all-cause dementia incidence in both age groups and with $\mathrm{AD}$ only in those under 75 years of age.

\section{Associations between sleep complaints, hypnotics, plasma $\beta$-amyloid levels, and incident dementia}

These analyses were implemented on a subgroup of 984 subjects with data available for plasma $A \beta$ measurements. These subjects were not different from the 5867 without these measurements: $60 \%$ of women, mean age of 73.6 ( \pm 5.2 ), $18.7 \%$ complained of EDS, $36.1 \%$ of DIS, $64.9 \%$ of DMS, $36.2 \%$ of EMA, and $22.7 \%$ were taking hypnotics at baseline and $9.9 \%$ were diagnosed dementia over the follow-up.

No association was found between baseline plasma $\mathrm{A} \beta_{42}, \mathrm{~A} \beta_{40}$, and its ratio (taken continuously or categorized into tertile) and (1) sleep complaints taken alone or combined, and hypnotic use at baseline, (2) and incident all-cause dementia and its subtypes.

\section{Discussion}

In this large cohort of community-dwelling elderly, EDS was associated with an increased risk of $21 \%$ for incident all-cause dementia and 58\% for DVC over 12 years, but no association was observed with AD incidence. Persistent use of hypnotics increased the risk for all-cause dementia and AD, but not DVC after adjustment for the depressive status. In contrast, no association was found between insomnia complaints, and SQ taken as individual factors or combined with EDS in the CSS score on the risk of incident dementia. We also reported that the association between hypnotic use and incident all-cause dementia and $\mathrm{AD}$ was stronger in diabetic subjects, with same trend in subjects below 75 years old for incident $\mathrm{AD}$. The association between EDS and all-cause dementia and DVC risk appeared greater in subjects over 75 years old.

EDS is a frequent condition in the elderly population. It may be due to multifactorial factors and may be associated with severe consequences [24, 25]. EDS is often comorbid with depression, poor sleep habits, obesity, cardiovascular disease, obstructive sleep apnea (OSA), 
and hypnotics intake, each of which being also associated with an increased risk of cognitive impairment [1, 26-28]. Prospective studies in older adults reported that EDS was independently associated with cognitive decline and dementia $[2,3,29]$. Our current results confirm and extend this finding in both all-cause dementia and DVC, within a larger sample of both men and women elderly persons over a long follow-up period, and with further adjustments particularly APOE- $\varepsilon 4$ status, cardiovascular, and metabolic diseases and depression. The mechanism underlying this association remains unclear. Self-reported EDS may be interpreted as a marker of sleep instability, with fragmented sleep and reduced slow-wave sleep, but also with OSA especially when it is severe. Recent data suggested that excessively sleepy OSA subtype has the greatest increased cardiovascular risk compared to other types of OSA [30]. Elderly subjects may lack of awareness of sleep apnea symptoms, but sleep apnea is associated with a higher risk of dementia and cardiovascular disorders [27, 31]. Several pathophysiological mechanisms may be involved such as disturbed slow-wave and rapid eye movement sleep, intermittent hypoxia, low-grade inflammation, oxidative stress, and brain $A \beta$ aggregation and tau hyperphosphorylation [32, 33]. Other studies also underlined the association between EDS, but not insomnia complaints, and higher risk of cardiovascular events $[34,35]$, the latter being potential mediators in the relationship between EDS and DVC incidence [3]. Here, we found that subjects with EDS over 75 years of age exhibited the highest risk of dementia, suggesting that EDS would be a predictor for late-onset age of dementia.

EDS in non-demented elderly subjects could also be associated with underlying neurodegeneration. A recent study found an association between EDS and global cortical thickness reduction primarily in regions with increased age-susceptibility which may indicate accelerated brain aging [36]. Degeneration of wakefulnesspromoting neurons in the cholinergic basal forebrain, noradrenergic locus coeruleus, and orexin hypothalamic systems may be damaged and cause sleep/wake abnormalities [37]. Thus, there are biologically plausible mechanisms that may explain these associations; however, further studies are required to explore these hypotheses.

Insomnia is one of the most prevalent sleep complaints in the elderly. We found no association between poor SQ, insomnia complaints and all-cause dementia, $\mathrm{AD}$ and DVC. Our results are in agreement with several studies reporting no association [2,12,38], but contrast with others showing the association between insomnia and dementia $[6,8,10]$. These inconsistencies across studies may be attributed to differences in methodology (adjustments, follow-up duration) and heterogeneity in cognitive and sleep assessment. Most of the positive associations between insomnia and dementia were not controlled for APOE- $\varepsilon 4$, depressive status, and the use of hypnotic $[6-8,10,33]$. To our knowledge, no previous studies evaluated the presence and severity of both daytime and nighttime sleep complaints in the elderly to assess for the potential additive role of sleep problems in the onset of dementia. Although not validated, we proposed to combine in the present study the five sleep complaints (EDS, insomnia complaints, and sleep quality) together in a global assessment called CSS; however, we found no association between this score and the onset of dementia risk.

Hypnotics (mainly BZD) at baseline were taken by almost a quarter of the subjects, who had more frequently EDS, insomnia complaints, and higher CSS score. Hypnotics use increased the risk of all-cause dementia and $\mathrm{AD}$ after several adjustments including depressive status. Moreover, hypnotic intake (especially for persistent users) was associated with incident all-cause dementia and $\mathrm{AD}$ in subjects below 75 years old and particularly in diabetic subjects. Few studies already underlined that $\mathrm{AD}$ and diabetes may share common pathophysiology, with prolonged excess of glucose leading to poor sleep quality and risk of neurodegeneration [39, 40]. The association between BZD use and dementia incidence was also reported [22, 41]; however, the biological mechanism that underlines this association is not well understood. Sleep is critical for ensuring brain metabolic homeostasis with large increases in the cortical interstitial space during slow-wave sleep compared to wake resulting in convective exchange between the cerebrospinal fluid (CSF) and the interstitial fluid [42]. The restorative function of sleep may therefore relate to increased clearance of potentially neurotoxic degradation products of neuronal activity that accumulate in the awake brain, such as $\mathrm{A} \beta$. With the chronic use of BZDs, the delta oscillations decrease, the glymphatic system dedicated to CSF transport, and metabolic waste drainage from the brain may be impaired resulting in $A \beta$ accumulation and deposition [43]. We report here no association between plasma $\mathrm{A} \beta_{40}-\mathrm{A} \beta_{42}$ levels and sleep complaints, with measurements performed at baseline therefore in subjects without dementia. These results were in line with a previous study including only men [8]. However, both studies measured $A \beta$ levels in the plasma, being less sensitive than CSF to estimate the impact of sleep disturbance on $A \beta$ kinetics. An increased longitudinal brain $A \beta$ accumulation assessed on a positron emission tomography was recently reported in elderly subjects with EDS and without dementia [44]. A proposed hypothesis suggests that EDS is a clinical manifestation of increased synaptic or network overload in the cingulate and precuneus regions, areas with high metabolic activity, and $\mathrm{A} \beta$ accumulation 
that may increase oxidative stress and neuronal death, and then the risk of AD.

The strengths of this study were the longitudinal design with a 12-year-follow-up, the large sample size, the low attrition rate, and the large number of potential predictors including socio-demographic, lifestyle, health characteristics, medications, and APOE- $\varepsilon 4$ status.

\section{Limitations}

This study had some limitations. Bias could have been introduced by the non-random exclusion of participants with missing data at baseline, who were older, had more comorbidities and sleep complaints and used more hypnotics; this may have underestimated the strengths of the associations reported. Although the prevalent demented subjects were excluded in this study, a potential reverse causality effect cannot be excluded. This phenomenon is inevitable in the study of subjects aged 65 and over because of the prodromal phase onset 10 to 20 years before symptoms. However, after the exclusion of incident demented subjects during the first 2 years of follow-up, the results remained unchanged. The possibility of over-adjustment could not be excluded: potential confounders with cardiovascular parameters could be intermediate variables in the pathway between sleep complaints, hypnotic intake, and dementia incident. Due to the exploratory nature of this study, correction for multiple comparisons were not made. Furthermore, the assessment of sleep complaints was only self-reported causing possible recall bias and a lack of accuracy in responses, although previous studies have assessed EDS with a similar question [29, 34, 35]; however, validated measures such as the Epworth Sleepiness Scale were not available in this study. In addition, we developed for the present study a clinical sleep severity (CSS) score to estimate the potential additive role of the five key sleep complaints on the risk of dementia; however, this CSS was only proposed for this present study and therefore has not been validated by other studies.

The drugs intake was verified by examining the prescriptions and medications themselves; however, duration, frequency, dose, and reason of the medication intake were not recorded. Regarding the duration of exposure to hypnotics, we performed 2 sensitivity analyses. First, we limited the study of the association between the use of hypnotics during the first 2 years and incident of dementia while maintaining a significant follow-up period (10 years). Then, we used time-dependent variable to consider hypnotics use throughout the followup. Both analyses showed that persistent use of hypnotic was associated with a higher risk of dementia. We were unable to directly measure symptoms of anxiety in this study. However, our models were adjusted for the CES-D score first designed to screen for depressive symptoms but also anxiety symptoms in the elderly population [45, 46]. The presence of sleep apnea was not evaluated; thus, the confounding effect of an underlying OSA syndrome in the relationship between EDS and the risk of dementia cannot be excluded. Unfortunately, sleep duration and objective measures of sleep and daytime sleepiness were not available for this study. Even difficult within the context of a large epidemiological studies, further studies using objective sleep tools are recommended. Finally, $\beta$-amyloid concentrations were assessed in the plasma instead of cerebrospinal fluid, being a less sensitive method, but still relevant to indicate individuals at shortterm risk of dementia or cognitive decline [22, 23].

\section{Conclusions}

EDS was associated with a high risk of all-cause dementia and even more strongly with DVC, but not with AD while the use of hypnotics was associated with incident all-cause dementia and AD. These results suggest that EDS and hypnotics intake may be targets for intervention strategies in dementia prevention. No association was found for insomnia complaints and quality of sleep assessed alone or combined with EDS after controlling for potential confounders. Further studies are required to elucidate the biological mechanisms involved in these associations and whether its management can prevent the risk of dementia.

\section{Abbreviations}

AD: Alzheimer's disease; APOE: Apolipropotein-e; BMI: Body mass index; BZD: Benzodiazepine; Cl: Confidence intervals; CSF: Cerebrospinal fluid; CSS: Clinical sleep score; DIS: Difficulty in initiating sleep; DMS: Difficulty maintaining sleep; DVC: Dementia with vascular component; EDS: Excessive daytime sleepiness; EMA: Early morning awakening; HR: Hazard ratio; OSA: Obstructive sleep apnea; SQ: Sleep quality.

\section{Acknowledgements \\ The authors acknowledge all participants in the 3-City Study.}

\section{Authors' contributions}

CC contributed to the conception and design of the study, to the analysis and interpretation of data, and wrote the first draft of the manuscript. CB and $\mathrm{CH}$ contributed in the acquisition of the data and revised the manuscript. AG revised the manuscript. IJ and YD contributed to the conception and design of the study, to the interpretation of data, and to the writing of the manuscript. The authors read and approved the final manuscript.

\section{Funding}

The Three-City Study is conducted under a partnership agreement between Inserm, the Victor Segalen - Bordeaux II University, and Sanofi-Synthélabo. The Fondation pour la Recherche Médicale funded the preparation and first phase of the study. The Three-City Study is also supported by the Caisse Nationale Maladie des Travailleurs Salariés, Direction Générale de la Santé, MGEN, Institut de la Longévité, Agence Française de Sécurité Sanitaire des Produits de Santé, the Regional Governments of Aquitaine, Bourgogne and LanguedocRoussillon and, the Fondation de France, the Ministry of Research-Inserm Programme "Cohorts and collection of biological material."The Lille Génopôle received an unconditional grant from Eisai. Part of this project is financed by 
two grants from the Agence Nationale de la Recherche (ANR) (projects 07 LVIE 004 and 06-PNRA-005) and Fonds de coopération scientifique Alzheimer (FCS 2009-2012).

\section{Availability of data and materials}

The datasets used and analyzed during the current study are available from the corresponding author on reasonable request.

\section{Declarations}

\section{Ethics approval and consent to participate}

The ethical committee of the Kremlin-Bicêtre University Hospital (France) approved the study protocol, and written informed consent was obtained from each participant.

\section{Consent for publication}

Not applicable.

\section{Competing interests}

$\mathrm{CC}, \mathrm{CB}, \mathrm{CH}, \mathrm{AG}$, and IJ declare that they have no competing interests. YD participated in advisory board for UCB Pharma, Jazz, Theranexus, Avadel, Idorsia, and Bioprojet, outside the submitted work.

\section{Author details}

1 Institute for Neurosciences of Montpellier INM, INSERM Montpellier, University of Montpellier, Montpellier, France. ${ }^{2}$ INSERM, Bordeaux Population Health Research Center, UMR1219, University of Bordeaux, Bordeaux, France. ${ }^{3}$ Memory Research and Resources Center, Department of Neurology, CHU Montpellier, Montpellier, France. ${ }^{4}$ National Reference Centre for Orphan Diseases, Narcolepsy- Rare Hypersomnias, Sleep Unit, Department of Neurology, CHU Montpellier, Service de Neurologie, Hôpital Gui-de-Chauliac, 80 avenue Augustin Fliche, 34295 Montpellier cedex 5, France.

Received: 13 September 2021 Accepted: 20 December 2021

Published online: 20 January 2022

\section{References}

1. Livingston G, Sommerlad A, Orgeta V, Costafreda SG, Huntley J, Ames $D$, et al. Dementia prevention, intervention, and care. Lancet Lond Engl. 2017:390(10113):2673-734.

2. Foley D, Monjan A, Masaki K, Ross W, Havlik R, White L, et al. Daytime sleepiness is associated with 3-year incident dementia and cognitive decline in older Japanese-American men. J Am Geriatr Soc. 2001:49(12):1628-32.

3. Elwood P, Bayer A, Fish M, Pickering J, Mitchell C, Gallacher J. Sleep disturbance and daytime sleepiness predict vascular dementia. J Epidemiol Community Health. 2011;65:820-4.

4. Merlino G, Piani A, Gigli GL, Cancelli I, Rinaldi A, Baroselli A, et al. Daytime sleepiness is associated with dementia and cognitive decline in older Italian adults: a population-based study. Sleep Med. 2010;11(4):372-7.

5. Tsapanou A, Gu Y, Manly J, Schupf N, Tang M-X, Zimmerman M, et al. Daytime sleepiness and sleep inadequacy as risk factors for dementia. Dement Geriatr Cogn Disord Extra. 2015;5(2):286-95.

6. Burke SL, Cadet T, Alcide A, O'Driscoll J, Maramaldi P. Psychosocial risk factors and Alzheimer's disease: the associative effect of depression, sleep disturbance, and anxiety. Aging Ment Health. 2018;22(12):1577-84.

7. Luojus MK, Lehto SM, Tolmunen T, Brem A-K, Lönnroos E, Kauhanen J. Self-reported sleep disturbance and incidence of dementia in ageing men. J Epidemiol Community Health. 2017;71(4):329-35.

8. Benedict C, Byberg L, Cedernaes J, Hogenkamp PS, Giedratis V, Kilander $L$, et al. Self-reported sleep disturbance is associated with Alzheimer's disease risk in men. Alzheimers Dement. 2015;1 1(9):1090-7.

9. Sindi S, Kåreholt I, Johansson L, Skoog J, Sjöberg L, Wang H-X, et al. Sleep disturbances and dementia risk: a multicenter study. Alzheimers Dement. 2018:1:14.

10. Yaffe K, Nettiksimmons J, Yesavage J, Byers A. Sleep quality and risk of dementia among older male veterans. Am J Geriatr Psychiatry. 2015;1:23.
11. Xu W, Tan C-C, Zou J-J, Cao X-P, Tan L. Sleep problems and risk of all-cause cognitive decline or dementia: an updated systematic review and metaanalysis. J Neurol Neurosurg Psychiatry. 2020;91(3):236-44.

12. Bokenberger K, Ström P, Dahl Aslan AK, Johansson ALV, Gatz M, Pedersen $\mathrm{NL}$, et al. Association between sleep characteristics and incident dementia accounting for baseline cognitive status: a prospective populationbased study. J Gerontol A Biol Sci Med Sci. 2017;72(1):134-9.

13. Lysen TS, Wolters FJ, Luik Al, Ikram MK, Tiemeier H, Ikram MA. Subjective sleep quality is not associated with incident dementia: the Rotterdam Study. J Alzheimers Dis JAD. 2018;64(1):239-47.

14. 3C Study Group. Vascular factors and risk of dementia: design of the Three-City Study and baseline characteristics of the study population. Neuroepidemiology. 2003;22(6):316-25.

15. McKhann G, Drachman D, Folstein M, Katzman R, Price D, Stadlan EM. Clinical diagnosis of alzheimer's disease: report of the NINCDS-ADRDA work group $\star$ under the auspices of department of health and human services task force on Alzheimer's disease. Neurology. 1984;34(7):939-44.

16. Román GC, Tatemichi TK, Erkinjuntti T, Cummings JL, Masdeu JC, Garcia $\mathrm{JH}$, et al. Vascular dementia: diagnostic criteria for research studies - report of the NINDS-AIREN international workshop. Neurology. 1993;43(2):250-60.

17. McKeith IG, Galasko D, Kosaka K, Perry EK, Dickson DW, Hansen LA, et al. Consensus guidelines for the clinical and pathologic diagnosis of dementia with Lewy bodies (DLB): report of the consortium on DLB international workshop. Neurology. 1996;47(5):1113-24

18. McKeith IG. Consensus guidelines for the clinical and pathologic diagnosis of dementia with Lewy bodies (DLB): report of the Consortium on DLB International Workshop. J Alzheimers Dis JAD. 2006;9(3 Suppl):417-23.

19. McKhann GM, Albert MS, Grossman M, Miller B, Dickson D, Trojanowski $J \mathrm{Q}$, et al. Clinical and pathological diagnosis of frontotemporal dementia: report of the Work Group on Frontotemporal Dementia and Pick's Disease. Arch Neurol. 2001;58(11):1803-9.

20. Dubois B, Burn D, Goetz C, Aarsland D, Brown RG, Broe GA, et al. Diagnostic procedures for Parkinson's disease dementia: recommendations from the Movement Disorder Society Task Force. Mov Disord. 2007;22(16):2314-24.

21. Dufouil C, Richard F, Fiévet N, Dartigues JF, Ritchie K, Tzourio C, et al. APOE genotype, cholesterol level, lipid-lowering treatment, and dementia: the Three-City Study. Neurology. 2005;64(9):1531-8.

22. Lambert J-C, Schraen-Maschke S, Richard F, Fievet N, Rouaud O, Berr C, et al. Association of plasma amyloid beta with risk of dementia: the prospective Three-City Study. Neurology. 2009;73(11):847-53.

23. Gabelle A, Richard F, Gutierrez L-A, Schraen S, Delva F, Rouaud O, et al. Plasma amyloid- $\beta$ levels and prognosis in incident dementia cases of the 3-City Study. J Alzheimers Dis JAD. 2013;33(2):381-91.

24. Gooneratne NS, Weaver TE, Cater JR, Pack FM, Arner HM, Greenberg AS, et al. Functional outcomes of excessive daytime sleepiness in older adults. J Am Geriatr Soc. 2003;51(5):642-9.

25. Ng WL, Shaw JE, Peeters A. The relationship between excessive daytime sleepiness, disability, and mortality, and implications for life expectancy. Sleep Med. 2018;43:83-9.

26. Bubu OM, Brannick M, Mortimer J, Umasabor-Bubu O, Sebastião YV, Wen Y, et al. Sleep, cognitive impairment, and Alzheimer's disease: a systematic review and meta-analysis. Sleep. 2017;1:40(1).

27. Yaffe K, Laffan AM, Harrison SL, Redline S, Spira AP, Ensrud KE, et al. Sleep disordered breathing, hypoxia, and risk of mild cognitive impairment and dementia in older women. JAMA J Am Med Assoc. 2011;306(6):613-9.

28. Lucchetta RC, da Mata BPM, Mastroianni P. de C. Association between development of dementia and use of benzodiazepines: a systematic review and meta-analysis. Pharmacotherapy. 2018;38(10):1010-20.

29. Jaussent I, Bouyer J, Ancelin M-L, Berr C, Foubert-Samier A, Ritchie K, et al. Excessive sleepiness is predictive of cognitive decline in the elderly. SLEEP. 2012;35(9):1201-7.

30. Pack Al, Magalang UJ, Singh B, Kuna ST, Keenan BT, Maislin G. Randomized clinical trials of cardiovascular disease in obstructive sleep apnea: understanding and overcoming bias. Sleep. 2020;9.

31. Gonzaga C, Bertolami A, Bertolami M, Amodeo C, Calhoun D. Obstructive sleep apnea, hypertension and cardiovascular diseases. J Hum Hypertens. 2015;29(12):705-12.

32. Baril A-A, Carrier J, Lafrenière A, Warby S, Poirier J, Osorio RS, et al. Biomarkers of dementia in obstructive sleep apnea. Sleep Med Rev. 2018;42:139-48. 
33. Pase MP, Himali JJ, Grima NA, Beiser AS, Satizabal CL, Aparicio HJ, et al. Sleep architecture and the risk of incident dementia in the community. Neurology. 2017;89(12):1244-50.

34. Blachier M, Dauvilliers Y, Jaussent I, Helmer C, Ritchie K, Jouven X, et al. Excessive daytime sleepiness and vascular events: the Three City Study. Ann Neurol. 2012;71(5):661-7.

35. Jaussent I, Empana J-P, Ancelin M-L, Besset A, Helmer C, Tzourio C, et al. Insomnia, daytime sleepiness and cardio-cerebrovascular diseases in the elderly: a 6-year prospective study. PloS One. 2013;8(2):e56048.

36. Carvalho DZ, St Louis EK, Boeve BF, Mielke MM, Przybelski SA, Knopman DS, et al. Excessive daytime sleepiness and fatigue may indicate accelerated brain aging in cognitively normal late middle-aged and older adults. Sleep Med. 2017;32:236-43.

37. Gabelle A, Gutierrez L-A, Jaussent I, Ben Bouallegue F, De Verbizier D, Navucet $S$, et al. Absence of relationship between self-reported sleep measures and amyloid load in elderly subjects. Front Neurol. 2019;10:989.

38. Blackwell T, Yaffe K, Ancoli-Israel S, Redline S, Ensrud KE, Stefanick ML, et al. Association of sleep characteristics and cognition in older community-dwelling men: the MrOS sleep study. Sleep. 2011;34(10):1347-56.

39. de la Monte SM. Type 3 diabetes is sporadic Alzheimer's disease: minireview. Eur Neuropsychopharmacol J Eur Coll Neuropsychopharmacol. 2014;24(12):1954-60.

40. Hanlon EC, Cauter EV. Quantification of sleep behavior and of its impact on the cross-talk between the brain and peripheral metabolism. Proc Natl Acad Sci. 2011;108(Supplement 3):15609-16.

41. Penninkilampi R, Eslick GD. A systematic review and meta-analysis of the risk of dementia associated with benzodiazepine use, after controlling for protopathic bias. CNS Drugs. 2018;32(6):485-97.

42. Xie L, Kang H, Xu Q, Chen MJ, Liao Y, Thiyagarajan M, et al. Sleep drives metabolite clearance from the adult brain. Science. 2013;342(6156):373-7.

43. Benveniste H, Heerdt PM, Fontes M, Rothman DL, Volkow ND. Glymphatic system function in relation to anesthesia and sleep states. Anesth Analg. 2019;128(4):747-58.

44. Carvalho DZ, St Louis EK, Knopman DS, Boeve BF, Lowe VJ, Roberts RO, et al. Association of excessive daytime sleepiness with longitudinal $\beta$-amyloid accumulation in elderly persons without dementia. JAMA Neurol. 2018;75(6):672-80

45. McQuaid JR, Stein MB, McCahill M, Laffaye C, Ramel W. Use of brief psychiatric screening measures in a primary care sample. Depress Anxiety. 2000;12(1):21-9.

46. Dozeman E, van Schaik DJF, van Marwijk HWJ, Stek ML, van der Horst HE, Beekman ATF. The center for epidemiological studies depression scale (CES-D) is an adequate screening instrument for depressive and anxiety disorders in a very old population living in residential homes. Int J Geriatr Psychiatry. 2011;26(3):239-46.

\section{Publisher's Note}

Springer Nature remains neutral with regard to jurisdictional claims in published maps and institutional affiliations.

Ready to submit your research? Choose BMC and benefit from:

- fast, convenient online submission

- thorough peer review by experienced researchers in your field

- rapid publication on acceptance

- support for research data, including large and complex data types

- gold Open Access which fosters wider collaboration and increased citations

- maximum visibility for your research: over 100M website views per year

At BMC, research is always in progress.

Learn more biomedcentral.com/submissions 